\section{How to become a generalist again}

$I^{f}$ $\mathrm{f}$ life seems like one inexorable process of specialization, take a closer look at the Dictyostelium slug. After forming from aggregated single cells, it is on its way to becoming a specialized fruiting body. But if its cells are dispersed and given nutrients they revert to their primitive, proliferative state. This dedifferentiation, say Mariko Katoh, Gad Shaulsky (Baylor College of Medicine, Houston, TX), and colleagues, is not a simple reversal of differentiation but a carefully regulated process. Similar regulation may ensure that dedifferentiating cells in a mammalian wound, for example, can fill in the wound without causing cancers or distorting the shape of the body part that was carefully crafted during development.
The group's claim is based on microarray results. Many of the transcriptional changes in dedifferentiating cells are a mirror image of those taking place in differentiating cells, but over 100 genes show changes specific to dedifferentiation. The set of genes is similar even when cells start dedifferentiating from different developmental stages.

One of the genes turned on during dedifferentiation encodes DhkA. Mutants lacking DhkA are slower to reinitiate cell division, but not DNA synthesis, during dedifferentiation. DhkA is a histidine kinase that, as part of a two-component system, is also required for the late differentiation event of sporulation. Shaulsky suggests that
Dedifferentiation

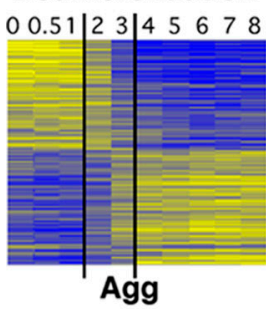

Development

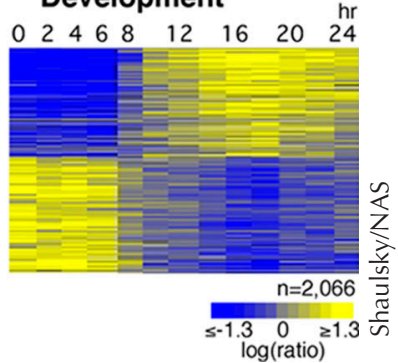

Although much of dedifferentiation (left) is the reverse of differentiation (right), other expression changes are specific to the process.

DhkA may be part of a checkpoint system in which completion of differentiation is contingent on accumulation of proteins (such as DhkA) necessary for dedifferentiation, thus ensuring that development is reversible.

Reference: Katoh, M., et al. 2004. Proc. Natl. Acad. Sci. USA. 101:7005-7010.

\title{
Metabolism as a production line
}

etabolic pathways might be smarter than we think, Vaccording to Alon Zaslaver, Uri Alon (Weizmann Institute, Rehovot, Israel), and colleagues. At least in bacterial amino acid biosynthesis pathways, the production schedules are designed using two principles that, according to theory, optimize the pathways for the fastest output using the least amount of enzymes.

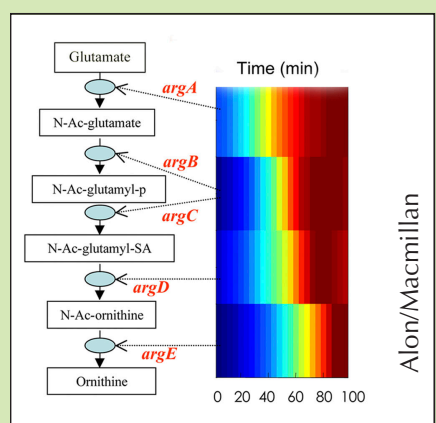

Promoters are activated only as they are needed.
"If the cell had an infinite amount of energy it would just dump very high levels of all these proteins together right away," says Alon. "But bacteria are limited by protein synthesis. In this economy they need to make tradeoffs."

Alon's group investigated the tradeoffs using 52 gene fusions to fluorescent proteins. They found that genes encoding early steps in a pathway are turned on both earlier and more aggressively, thus ensuring that later gene products have a sufficiently high concentration of substrate on which to act. A computer program designed to optimize production in a mathematical model of a similar pathway came up with a strategy that had the same two dynamic principles.

The "just in time" approach-ordering transcription based on when the gene products are used-has been seen in pathways controlling development and phage and flagellum assembly. For the flagellum case, others have shown that the ordering correlates with the varying affinity of a single transcription factor; in an upcoming issue of Cell, Alon's team reports that the order can be changed with simple point mutations in the flagellum promoters.

Reference: Zaslaver, A., et al. 2004. Nat. Genet. 36:486-491.

\section{Making waves}

Waves of activity need some help in propagating through a cell cycle, according to Attila Becskei, Monica Boselli, and Alexander van Oudenaarden (MIT, Cambridge, MA).

Each transcriptional wave from a yeast cell cycle promoter lasts 20-25 min; so stringing together 3 or 4 of them should allow the construction of a simple 90-min cell cycle. But, says van Oudenaarden, "if you didn't optimize the system, the cell cycle waves get washed out very easily. Within a quarter or a third of a 90-minute cycle, the waves are almost completely gone."

Rather than investigating every last detail of real cell cycle oscillators, the MIT group built, both in yeast cells and in silico, a simple circuit that transmits cell cycle-like oscillations. They found that a slow process such as

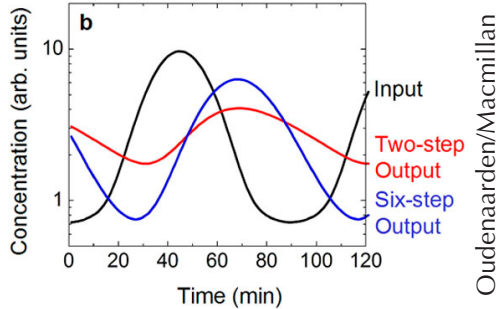

Multiple shorter steps are better at preserving oscillations. transcription (and the lengthy persistence of the resulting mRNA) led to a loss of the initial periodicity information from a cell cycle promoter.

A faster process, such as accelerated nuclear import, gave a shorter delay but maintained the high peak-to-trough information of the original oscillation. Stringing together a series of such fast processes, such as protein modifications, should give accurate oscillations of approximately the correct duration. The high peak-to-trough ratio can also be restored with feedback loops and nonlinear activation and degradation steps. All of these tricks were known to exist in the cell cycle, but the MIT group has now established why they are necessary.

Reference: Becskei, A., et al. 2004. Nat. Cell Biol. 6:451-457. 